\title{
A Qualitative Study of Anticipated Decision Making around Type 2 Diabetes Genetic Testing: the Role of Scientifically Concordant and Discordant Expectations
}

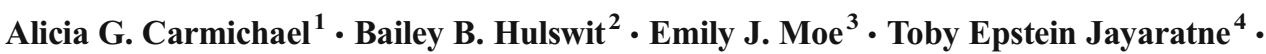 \\ Beverly M. Yashar ${ }^{5}$
}

Received: 3 August 2015 / Accepted: 13 July 2016/Published online: 28 July 2016

(C) National Society of Genetic Counselors, Inc. 2016

\begin{abstract}
Type 2 diabetes mellitus (T2DM) genetic testing is undergoing clinical trials to measure the efficacy of genetic counseling for behavior-based risk reduction. The expectations patients bring to the testing process may play an important role in individual outcomes. We conducted a qualitative exploration of anticipated decision-making and expectations around T2DM genetic testing. Semi-structured interviews were completed with Mexican Americans $(n=34)$, nonHispanic Black Americans $(n=39)$, and non-Hispanic White Americans ( $n=39)$ at risk for T2DM. Transcripts were analyzed for themes. Most participants would accept T2DM genetic testing in order to motivate risk-reducing behaviors or apprise family members of their risk. Participants who would decline testing wished to avoid emotional distress or believed the test would not reveal new risk information. Non-Hispanic Whites and those with college education declined genetic testing more often than other groups. Those without college education were more likely to have testing expectations that were discordant with current science, such as conflating genetic
\end{abstract}

Alicia G. Carmichael

almagior@umich.edu

1 BioSocial Methods Collaborative, Research Center for Group Dynamics, Institute for Social Research, University of Michigan, 426 Thompson St, Ann Arbor, MI 48109-1248, USA

2 Center for Molecular Medicine and Genetics, Wayne State University, Detroit, MI, USA

3 Genetics and Genomics Program, Children's Hospital of Wisconsin, Milwaukee, WI, USA

4 Department of Health Behavior and Health Education, School of Public Health, University of Michigan, Ann Arbor, MI, USA

5 Department of Human Genetics, University of Michigan, Ann Arbor, USA testing with common 'blood tests.' Understanding expectations and decision-making factors around T2DM genetic testing will better prepare healthcare professionals to counsel their patients. This may lead to a higher efficacy of T2DM genetic testing and counseling.

Keywords Diabetes · Genetic testing · Decision-making · Qualitative research

The global burden of diabetes is responsible for more than $\$ 612$ billion in healthcare costs and a loss of over 34.6 million years of life due to premature death per year (International Diabetes Federation, 2013; WHO Department of Health Statistics and Information Systems, 2013). Several clinical trials have demonstrated that behavioral intervention can reduce the incidence of type 2 diabetes mellitus (T2DM; reviewed in Schellenberg et al., 2013); however, large-scale implementation has proven challenging across cultures and within existing healthcare systems, resulting in minimal effect on T2DM prevalence (Cardona-Morrell et al., 2010; Yates et al., 2009). Thus, researchers continue to pursue new ways of augmenting behavioral interventions and new methods of targeting individuals at high risk for T2DM. Genetic testing and genetic counseling for T2DM are being pursued in the hopes that they may aid in the identification of individuals at higher risk for T2DM and motivate risk-reducing lifestyle modifications.

Several clinical trials (Centre d'Etudes et de Recherche pour l'Intensification du Traitement du Diabète, 2012; David Grant U.S. Air Force Medical Center, Duke University, 2013; Proove Bioscience, Inc, 2015) and other studies have already been initiated to measure the clinical value of T2DM genetic/ familial risk assessment and to develop best practices around genetic counseling (Grant et al., 2009; Mills, Powell, Barry \& 
Haga, 2015; Nishigaki et al., 2007; Nishigaki et al., 2011; Nishigaki et al., 2014; Vassy et al., 2013; Voils et al., 2015). To date, the results of these studies have been discouraging, concluding that T2DM genetic testing as it currently exists is not a promising clinical tool. Nevertheless, genetic medicine is ever evolving. It is proper and necessary that research into the human factor of genetic testing continue to advance even, and preferably, ahead of the science of genetics itself. In this qualitative paper, we will further explore T2DM genetic testing and genetic counseling from the lay public's perspective. Our study will provide insight on the expectations, concerns, and decision-making factors that patients may bring to an initial genetic counseling and testing appointment. We impose very little suggestion on our participants about what form T2DM genetic testing may take. Instead, they supply their own framework based on their own knowledge - a framework which would serve as a starting point for any clinical encounter and thus one which may be of interest to clinicians.

T2DM, like other common multifactorial disorders, poses a challenge to traditional genetic counseling paradigms (Becker et al., 2011; O'Daniel, 2010; Wright \& Kroese, 2009). There are some commonalities between T2DM genetic testing and genetic testing for rare Mendelian disorders. Both share three major opportunities around which counseling could be incorporated: risk assessment, decision-making, and results disclosure. Unlike rare Mendelian disorders, however, SNP-based genetic testing for T2DM produces complex results that, at this time, only modestly enhance risk assessment beyond traditional risk measures (the difference between a "high" phenotypic risk estimate and a combined "high" genetic plus phenotypic risk estimate is approximately $6 \%$; Almgren et al., 2011; de Miguel-Yanes et al., 2011; Meigs et al., 2008; Waxler et al., 2012). In the case of T2DM genetic testing, the additional challenge to counselors and patients comes from grappling with the paradox of complex-yet-limited genetic information and the difficulty of weaving this discussion throughout each of the three major counselling opportunities.

Because the clinical utility of T2DM genetic testing is still under investigation, research on T2DM genetic testing and counseling has been primarily outcome-oriented rather than process-oriented. In other words, what limited research exists has focused on psychological and behavioral reactions to genetic results disclosure, rather than preceding processes, such as decision-making. The only largescale clinical trials completed to date have failed to demonstrate a statistically significant effect of T2DM genetic testing and counseling on lifestyle modification (Grant et al., 2013; Voils et al., 2015). Although several studies using hypothetical scenarios initially raised the concern that low T2DM genetic risk results would decrease patients' motivation to improve lifestyle, (Grant et al., 2009; Vassy et al., 2013; Vassy et al., 2012) neither positive nor negative differences in motivation or behaviors have been detected among patients receiving high versus low genetic risk results (Grant et al., 2013; Voils et al., 2015).

Several factors may account for the apparent lack of utility in T2DM genetic testing and counseling. Although heritability estimates suggest that T2DM genetic tests could be further improved (Almgren et al., 2011), the difference between "low" and "high" genetic risk results is currently less than $10 \%$ (Waxler et al., 2012). Additionally, many potential confounders of the relationship between genetic risk results and psychological and behavioral outcomes have yet to be considered (e.g., patients' decision-making process and expectations about genetic testing; Klitzman, 2010). Thus, it is important to take a step back and examine the public's preconceptions about T2DM genetic testing in order to identify other factors that may play into the efficacy of the test and to better prepare clinicians to support their patients throughout the testing and counseling process.

As a first step towards this goal, we conducted an exploratory qualitative study of anticipated genetic testing decisionmaking among individuals at risk for T2DM using hypothetical scenarios. We also only included individuals who indicated in a previous structured interview that they believed in a genetic contribution to their risk for T2DM. Based on feedback from interview pretesting, we determined that participants who did not already believe in a genetic contribution to their T2DM would be unable to suspend their disbelief and would have thus been unable to respond to the hypothetical scenario. Because the health burden of T2DM is unequally distributed across race/ethnicity (American Diabetes Association [ADA], 2015; U.S. Department of Health and Human Services, 2008), we examined three U.S. subpopulations: Mexican Americans, non-Hispanic Blacks, and nonHispanic Whites. Participants were asked to decide whether they would choose to have genetic testing related to T2DM and then prompted to explain their decision. Themes around decision-making and testing expectations were evaluated in light of demographic background and participants' prior risk perceptions.

\section{Methods}

\section{Participants}

The Genetic Explanations for Type 2 Diabetes: Prevention Implications (GEDI) project is a national, landline telephone survey of non-diabetic Mexican Americans, non-Hispanic Blacks, and non-Hispanic Whites aged 18 to 75 who live in the contiguous U.S. GEDI used mixed quantitative and qualitative methodology. Phase I of GEDI employed a structured survey (conducted August 2011 through February 2012). In Phase II, a subset of Phase I participants were recontacted to 
complete a semi-structured qualitative interview (conducted February through April 2012). This paper primarily discusses data from Phase II. The study was approved by the University of Michigan's Institutional Review Board (\# HUM00058581). Oral informed consent was obtained from all participants.

Phase I participants were recruited using randomly generated landline numbers (Marketing Systems Group). During Phase I, self-reported risk factors for T2DM and beliefs about the causes of T2DM were assessed. Phase I participants $(N=1168)$ were then purposively sampled for recruitment into Phase II $(n=112)$ based on two eligibility criteria: (1) at-risk status for T2DM and (2) their belief that their personal genetics influenced their risk for developing T2DM (in order to avoid problematic failure to suspend disbelief, as described in the Introduction). At Phase I, genetic beliefs were assessed by asking "In your opinion, do your genes increase, decrease, or have no influence on your chance of getting type 2 diabetes?" Those who believed their genes had no influence were ineligible for Phase II. Overall, 475 Phase I participants (41\%) met Phase II eligibility criteria; two hundred twentyfive Phase I telephone lines were released for Phase II recruitment.

Participants who reported prediabetic status or having received high-risk warning from their physicians were considered at-risk for T2DM. To determine at-risk status for participants without prediabetes or a physician's warning, self-reported risk factors were compared to a subset of ADA screening criteria (ADA, 2015). Participants were classified as at-risk if they were overweight $(\mathrm{BMI} \geq 25)$ and had at least one other risk factor: severe obesity (BMI $\geq 35$ ), physical inactivity (U.S. Department of Health and Human Services, 2008), a first-degree relative with diabetes, a history of gestational diabetes, high blood pressure, high cholesterol, heart disease, or selfidentification with a high-risk race/ethnicity (non-Hispanic Black or Mexican American).

\section{Interviews}

Telephone interviews $(M=26.5 \mathrm{~min} ; S D=7.87 \mathrm{~min})$ were conducted by six professional interviewers from the University of Michigan Institute for Social Research. Five of the interviewers were women and one was a man. One interviewer identified as non-Hispanic Black, while the other five identified as non-Hispanic White. Two interviewers were bilingual Spanish/English speakers. Interviewer education ranged from Associates through Bachelor's degrees from a variety of fields. All interviewers were trained under the Institute for Social Research's General Interviewing Technique program and received study-specific training, which included instruction and certification in the use of computer-aided telephonic interviewing tools, demeanor, and open-ended interview techniques. All interviewers completed mock interviews during training, with periodic quality checks during data collection.

This paper analyzes responses to the following interview questions:

Suppose there was a test to see if your genes increase your chance of getting type 2 diabetes.

1 Would you want to get tested?

2 Why would you (not) want to get tested? / Are there any reasons why you would (not) want to get tested?

In order to identify decision-making factors originating from the participants themselves, they were not provided information about the sensitivity or the risks/benefits of testing. In order to further explore their decision-making process, participants who focused only on barriers to genetic testing, such as a fear of needles, were probed to explain what their decision would be if such barriers did not exist.

\section{Data Analysis}

NVivo (version 10; QSR International, MA, USA) was used for transcript coding. Stata (version 12; StataCorp LP, TX, USA) was used to calculate descriptive statistics of demographic data, frequencies of themes, and inter-coder reliability. Due to a scarcity of literature on perceptions about genetic testing related to T2DM, transcribed interviews were analyzed through conventional content analysis using inductive code generation as described by Hsieh and Shannon (2005). An inductive, data-driven codebook was developed through an iterative process of open-coding and code refinement until theoretical saturation was achieved (MacQueen et al., 1998). All authors and coders participated in the iterative code development process. Coders received training in qualitative methods from authors A.G.C, T.E.J., and B.M.Y., including a review of qualitative literature and practice coding sessions. Codebook training and practice coding continued until an overall Cohen's kappa of 0.7 was reached. The value 0.7 was chosen a priori as a conservative measure of inter-coder reliability within the context of exploratory research (Lombard et al., 2002). Each transcript was then independently coded by two coders drawn from a team of four (including E.J.M. and B.B.H.). Inter-coder reliability was continually assessed throughout the coding process to ensure coding consistency was maintained, with periodic codebook review sessions. All coders were non-Hispanic White women. Two coders were Master's students, one of whom was studying Genetic Counseling and the other was studying Health Behavior and Health Education. The other two coders were Bachelor's students, one of whom was studying Genetics and Ethics and the other was studying Psychology. All transcripts 
were reviewed by at least one Master's student. Coders resolved coding disagreements through discussion.

\section{Results}

Demographics, risk perceptions, and secondary T2DM risk factors for the 112 participants are presented in Table 1 . Unsurprisingly, due to the ADA risk factor inclusion criteria, the most common T2DM risk factor reported by all subsamples was being overweight (BMI $\geq 25$ ). Mean BMI was similar among Mexican American $(M=30.8, S D=5.87)$, Black $(M=32.3, S D=6.79)$, and White $(M=31.2, S D=5.22)$ participants. Excluding race/ethnicity, the most frequently reported secondary risk factor for T2DM was family history among Mexican Americans (38\% of participants), high blood pressure among non-Hispanic Blacks (56\%), and physical inactivity and high blood pressure among non-Hispanic Whites (both $49 \%$ ). Despite these self-reported T2DM risk factors, $44 \%$ of all participants believed their risk was low.

\section{Anticipated Genetic Testing Decision}

Overall, most participants $(n=91)$ anticipated utilizing a T2DM genetic test if one were offered to them. A much smaller portion $(n=18)$ would decline. Very few participants $(n=2)$ were unable to make a final decision. No testing decision could be determined for one participant due to interviewer error. Importantly, participants with high-perceived risk for T2DM were more likely to reject genetic testing $(n=7,23 \%)$ than those with average $(n=3,9 \%)$ or low $(n=8,16 \%)$ risk perceptions. Non-Hispanic White participants rejected testing more frequently $(n=8,21 \%)$ than Mexican Americans $(n=4$, $11 \%$ ) or non-Hispanic Blacks ( $n=6,15 \%)$. Participants with college education anticipated declining genetic testing more frequently $(n=16,20 \%)$ than those with less education $(n=2$, $7 \%$ ). Statistical testing is inappropriate given our qualitative design, and the intention of this study is neither to establish definitive group differences nor to generalize our conclusions to the wider U.S. population. The data suggest, however, a potential pattern between education, race/ethnicity, and

Table 1 Demographics, risk perception, and risk factors by race/ethnicity, $n(\%)$

\begin{tabular}{|c|c|c|c|}
\hline Characteristic & $\begin{array}{l}\text { Mexican Americans } \\
(n=34)\end{array}$ & $\begin{array}{l}\text { Non-Hispanic Blacks } \\
(n=39)\end{array}$ & $\begin{array}{l}\text { Non-Hispanic Whites } \\
(n=39)\end{array}$ \\
\hline \multicolumn{4}{|l|}{ Gender } \\
\hline Women & $17(50)$ & $21(54)$ & $20(51)$ \\
\hline Men & $17(50)$ & $18(46)$ & $19(49)$ \\
\hline \multicolumn{4}{|l|}{ Age } \\
\hline $18-44$ & $20(59)$ & $12(31)$ & $11(28)$ \\
\hline $45-60$ & $11(32)$ & $17(44)$ & $14(36)$ \\
\hline $61-75$ & $3(9)$ & $10(26)$ & $14(36)$ \\
\hline \multicolumn{4}{|l|}{ Education } \\
\hline No college & $11(32)$ & $11(28)$ & $8(21)$ \\
\hline College & $23(68)$ & $28(72)$ & $31(79)$ \\
\hline \multicolumn{4}{|l|}{ Annual income } \\
\hline$\leq \$ 25,000$ & $4(12)$ & $7(18)$ & $7(18)$ \\
\hline$\$ 25,001-\$ 50,000$ & $15(44)$ & $12(31)$ & $6(15)$ \\
\hline$\$ 50,001-\$ 85,000$ & $7(21)$ & $9(23)$ & $6(15)$ \\
\hline$>\$ 85,000$ & $6(18)$ & $10(26)$ & $16(41)$ \\
\hline \multicolumn{4}{|l|}{ Perceived T2DM risk } \\
\hline Low & $18(53)$ & $13(33)$ & $18(46)$ \\
\hline Average & $8(24)$ & $14(36)$ & $10(26)$ \\
\hline High & $8(24)$ & $12(31)$ & $11(28)$ \\
\hline \multicolumn{4}{|l|}{ Secondary T2DM risk factors } \\
\hline First-degree relative with diabetes & $13(38)$ & $17(44)$ & $13(33)$ \\
\hline Heart disease & $0(0)$ & $3(8)$ & $6(15)$ \\
\hline High blood pressure & $9(27)$ & $22(56)$ & $19(49)$ \\
\hline High cholesterol & $10(29)$ & $17(44)$ & $16(41)$ \\
\hline Physical inactivity & $11(32)$ & $13(33)$ & $19(49)$ \\
\hline Severe obesity & $6(19)$ & $13(33)$ & $7(18)$ \\
\hline
\end{tabular}

Percentages may not sum to 100 due to rounding or exclusion of missing data 
anticipated testing decisions (Table 2). Among non-Hispanic Blacks and Whites, but not among Mexican Americans, those with college education tended to have decreased interest in genetic testing.

\section{Theme Development and Concordance Analysis}

Codes were collapsed into higher-level themes organized around two related topics, elements of the decision-making process and genetic testing expectations. Three major themes emerged from participant's decision-making explanations: Information Orientation, Barriers to Testing, and Relevancy Based on Age. By "orientation" we mean attitudes towards genetic information. Information Orientation was by far the most frequent and rich overarching theme, warranting further segmentation based on participants' level of interest or ambivalence towards the content of genetic test results, as follows: Information-Seeking, Information-Avoiding, and Information-Neutral. We use the term "barrier" broadly to mean all things peripheral to the genetic test result itself, whether physical or psychological, which would prevent an individual from obtaining an otherwise desirable test.

Participants' expectations around genetic testing were also summarized by three major themes: Testing Procedure, Knowledge about Genetics, and Content of Test Results. Participants' expectations were compared with existing T2DM genetic counseling protocols (Grant et al., 2013; Waxler et al., 2012). Expectations were categorized as concordant with that of genetics professionals if they aligned with the following information, and were categorized as discordant if they did not:

- $\quad$ TTesting Procedure] Genetic testing procedures are typically minimally invasive and low-risk, including blood draws, blood spots, cheek scrapes, or saliva samples.

- [Knowledge about Genetics] While genetic tests may be refined, the underlying genetic information is permanent and does not change across the life course.
- [Knowledge about Genetics] Genetic variants do not "skip" generations.

- [Content of Test Results] Genetic test results related to T2DM provide information based exclusively on genes. Some researchers have attempted to combine genetic and lifestyle risk factors for a more holistic, individualized risk report (Almgren et al., 2011; de Miguel-Yanes et al., 2011; Grant et al., 2013; Meigs et al., 2008; Waxler et al., 2012), but methods for the combination of these results have not yet been fully developed.

- [Content of Test Results] T2DM-related genetic information is predictive and not diagnostic.

Concordance and discordance were not treated as binary opposites. Therefore, percent concordance and percent discordance will not sum to 100 . A single participant could demonstrate concordance, discordance, both, or, if there was insufficient evidence, neither concordance nor discordance. Participants whose responses provided insufficient evidence for concordance or discordance tended to be terse or vague despite probing and encouragement from the interviewer. For example,

Interviewer:Ifyou did get tested, what do you think your
test results might show?
Respondent:I have no idea.
Interviewer: What do you think? Given on what you told
me about-what we've talked about so far, what do you
think your test results might show?
Respondent:It may show more than I have an idea.

\section{Elements of the Decision Making Process}

Participants were asked to explain both their reasons against and in favor of T2DM genetic testing. These themes and their subthemes are summarized below. Illustrative quotations are presented in Table 3.

Table 2 Relationships between race/ethnicity and education among themes

\begin{tabular}{|c|c|c|c|c|c|}
\hline \multirow[t]{2}{*}{ Race/Ethnicity } & \multirow[t]{2}{*}{ Education $(n)$} & \multicolumn{2}{|c|}{ Testing decision $n(\%)$} & \multirow[t]{2}{*}{ Concordant statements $n(\%)$} & \multirow[t]{2}{*}{ Discordant statements $n(\%)$} \\
\hline & & Yes & No & & \\
\hline \multirow[t]{2}{*}{ Mexican American } & No College (11) & $10(91)$ & $1(9)$ & $6(55)$ & $8(73)$ \\
\hline & College (23) & $20(87)$ & $3(13)$ & $17(74)$ & $7(30)$ \\
\hline \multirow[t]{2}{*}{ Non-Hispanic Black } & No College (11) & $11(100)$ & $0(0)$ & $4(36)$ & $8(73)$ \\
\hline & College (28) & $22(79)$ & $6(21)$ & $22(79)$ & $9(32)$ \\
\hline \multirow[t]{2}{*}{ Non-Hispanic White } & No College (8) & $7(88)$ & $1(13)$ & $6(75)$ & $3(38)$ \\
\hline & College (31) & $21(68)$ & $7(23)$ & $22(73)$ & $6(20)$ \\
\hline
\end{tabular}

Percentages may not sum to 100 due to rounding or exclusion of missing data. Two participants' testing decisions were classified as "Maybe - neither yes nor no." No testing decision was given by one non-Hispanic White, college-educated participant due to interviewer error. These participants' data are omitted from testing decision 
Table 3 Themes and subthemes with representative quotations

\begin{tabular}{|c|c|}
\hline Theme & Quotations \\
\hline \multicolumn{2}{|c|}{ Elements of the decision-making process } \\
\hline Information-seeking & $\begin{array}{l}\text { Sometimes when it's four o'clock and I'm exhausted } \\
\text { and I don't want to go to the gym- - that [my genetic risk] } \\
\text { might be one more reason in the back of my head that } \\
\text { I do make that effort to go. }\end{array}$ \\
\hline Information-avoiding & $\begin{array}{l}\text { I'm not ready to go ahead and switch over to eating that healthy. And I feel like if I know that [I have genetic risk], then I'll } \\
\text { be bound } \\
\text { by eating proper the whole time and I-I really just gotta get right. }\end{array}$ \\
\hline Information-neutral & $\begin{array}{l}\text { I mean I know my family history, I know what I need to do, I know } \\
\text { what could possibly put me at greater risk. Therefore, no, } \\
\text { I don't need to be tested. }\end{array}$ \\
\hline Barriers to testing & $\begin{array}{l}\text { You got a } \$ 6000 \text { bill just from going to get tested and you don’t } \\
\text { even have money to buy groceries. }\end{array}$ \\
\hline Relevancy based on age & $\begin{array}{l}\text { I'd personally probably wouldn't simply because of my age ... } \\
\text { I've kind of become set in my ways. }\end{array}$ \\
\hline \multicolumn{2}{|l|}{ Genetic testing expectations } \\
\hline Content of test results & $\begin{array}{l}\text { Discordant: It's [the test is] gonna show you if you have the } \\
\text { diabetes or the risk. ... Maybe it's going to show me how my } \\
\text { cholesterol is in the moment, my ascorbic acid, my blood, } \\
\text { my white cells and my red cells in my body, in my blood. } \\
\text { Concordant: I mean you're not just talking about lab results that } \\
\text { looking at how well your body is functioning. You're not } \\
\text { talking about it-you're talking about genes specifically, right? }\end{array}$ \\
\hline $\begin{array}{l}\text { Knowledge about } \\
\text { genetics }\end{array}$ & $\begin{array}{l}\text { Discordant: They say it [the gene] skips a generation, but I know } \\
\text { it didn't with my brother. My mother has it [diabetes]. } \\
\text { Concordant: Well because-one thing is because I can't change } \\
\text { that part of it [genetic risk]. If you know the things that predispose you, } \\
\text { if you know that and if you're willing to work to minimize-some things } \\
\text { you're never going to be able to change. }\end{array}$ \\
\hline Testing procedure & $\begin{array}{l}\text { Discordant: I don't know how they do the test. But if it was some test that was } \\
\text { dangerous where they had to open you up and take your heart out and take blood } \\
\text { out of the heart or something and sew you back up, then I would probably think } \\
\text { harder about it. } \\
\text { But if they just had to take a blood test and then I would see no problem. } \\
\text { Concordant: [...] I guess if there was a free [genetic] test at a clinic and they } \\
\text { pricked my blood, } \\
\text { you know what I mean, if it was a simple thing to just go do. }\end{array}$ \\
\hline
\end{tabular}

Information Orientation ( $\boldsymbol{n}=\mathbf{8 8}$ ) Participants' orientation toward the information provided by genetic testing was central to most decision-making explanations. Information Orientation included three subthemes: Information-Seeking $(n=75)$, Information-Avoiding $(n=16)$, and InformationNeutral $(n=14)$. Some information-seeking participants believed that collecting their genetic information was good for its own sake ( $n=12,16 \%)$, but most $(n=63,84 \%)$ designated at least one practical application for their test results: to identify risk-reduction strategies, increase motivation to take action, to raise awareness of T2DM risk among their family members, and/or to diagnose T2DM. Information-avoiding participants believed the test results could provoke psychological burdens such as unwanted pressure to change one's lifestyle and stress over the potential for disease. Informationneutral participants described T2DM genetic testing as providing no added value because of the impossibility of modifying genetic risk factors directly or because they were already aware of their phenotypic or family history-based risk status.

Barriers to Testing $(\boldsymbol{n}=\mathbf{2 1})$ Examples of barriers to testing included the expense of the test, unpleasant testing process, and lack of confidentiality. Participants explained that a highcost test, particularly one not covered by insurance, would be a luxury when weighed against more immediate needs. Concern about an unpleasant testing process ranged from fear that the test would involve a dangerous or extremely painful medical procedure, to the common phobia of needles, to the more mild worry that the testing process would be "a hassle." Participants who discussed confidentiality worried that 
insurers would discriminate against them based on their test results.

Relevancy Based on Age $(\boldsymbol{n}=\mathbf{1 0})$ Some participants indicated that the decision to test should be based on one's age. The definition of "right" versus "wrong" age varied and was described with respect to life stage or relative to the participant's own age. The concept of "right age" was tied to the belief that this was an age at which (1) health habits were still malleable, (2) preventive actions were still likely to be effective, and/or (3) risk for T2DM was increasing.

\section{Genetic Testing Expectations}

In justifying their anticipated testing decisions, participants discussed their expectations about the test. As described in Methods, these expectations centered around three major themes and were further categorized as concordant or discordant with the standard medical procedures. While most cases could be coded as containing concordant and/or discordant statements $(n=99)$, some responses were unclassifiable $(n=13)$ due to either ambiguity or absence of content.

Content of Test Results $(\boldsymbol{n}=\mathbf{8 7})$ Most of the participants who discussed the content of their test results made concordant statements ( $n=64,74 \%$ of cases), such as expecting the test to provide risk information, understanding the exclusively genetic nature of the test, and believing in the stability of their genetic information. Discordant expectations around the content of test results were generally subtle. Over a third $(n=35$, $40 \%$ ) of participants who discussed the content of their test results conflated genetic test results with routine "blood tests" related to T2DM risk (e.g., lipid panels, fasting plasma glucose screening), believed the genetic test results would in some way account for current physical and dietary behaviors, or believed the test would be diagnostic rather than predictive.

Knowledge about Genetics $(\boldsymbol{n}=54)$ Participants' genetic beliefs were more often concordant with genetics professionals $(n=41,37 \%)$ than discordant $(n=15,13 \%)$. Participants demonstrating concordant genetic beliefs discussed, for example, the link between their test results and inherited risk factors faced by family members. Participants demonstrating discordant genetic beliefs anticipated that their test results might change over time. Others discussed "skipped generation" inheritance patterns, which, if conceived of as originating in the genotype, are common misconceptions arising when diseases exhibit X-linked inheritance, imprinting, or reduced penetrance (Klitzman, 2010; Tessaro et al., 2005).

Testing Procedure $(\boldsymbol{n}=\mathbf{6})$ All participants $(n=6)$ who discussed the genetic testing process made at least one statement suggesting an understanding of the genetic testing process that was concordant with actual medical protocols; however, three of these participants were also uncertain about the testing process and believed the test was at least potentially invasive or dangerous.

\section{Relationship between Anticipated Decisions, Decision Making Factors, Genetic Testing Expectations, and Background Factors}

Decision-making themes varied by testing decision, education, race/ethnicity, and perceived risk (Table 4). Unsurprisingly, those who anticipated declining genetic testing, and those with characteristics suggested to be associated with declining genetic testing (college education, nonHispanic White identity, high perceived risk), tended to focus on Information-Neutrality and Information-Avoiding subthemes, rather than Information-Seeking. Non-Hispanic Whites also cited Barriers to Testing more often than Mexican American or non-Hispanic Black participants, though these barriers tended to be psychological rather than physical or financial in nature. Similar to the pattern observed between race/ethnicity, education, and anticipated testing decision, the data suggest a potential relationship between concordance, discordance, and education, and race/ethnicity (Table 2). While those with higher education tended to have a higher frequency of concordant statements overall, there was virtually no difference in the frequency of concordant statements between non-Hispanic Whites with and without a college education.

\section{Discussion}

We interviewed 112 Mexican Americans, non-Hispanic Blacks, and non-Hispanic Whites who had significant risk factors for T2DM about their desire for a hypothetical genetic test related to T2DM. Our qualitative analysis revealed findings that reinforce and augment existing research in several ways. In agreement with previous research (Grant et al., 2009; Haga et al., 2013; Markowitz et al., 2011), the vast majority of our participants believed they would accept genetic testing for T2DM risk if it was offered to them. We further identified two potential decision-making trends: (1) those who identified as non-Hispanic White and (2) those with college-level education (except Mexican Americans) were more likely to reject or feel hesitant about T2DM genetic testing compared to other groups.

The purpose of this qualitative research was exploratory; it was not designed to support statistical comparisons. Further investigation in a representative national sample is necessary to investigate potential group differences or interactions. If the trends we observed were to be confirmed, our qualitative 
Table 4 Frequency of decision-making themes and subthemes by participant characteristics, $n(\%)$

\begin{tabular}{|c|c|c|c|c|c|}
\hline \multirow[t]{3}{*}{ Characteristic $(n)$} & \multicolumn{5}{|c|}{ Themes and subthemes } \\
\hline & \multicolumn{3}{|c|}{ Information orientation } & \multirow[t]{2}{*}{ Barriers to testing } & \multirow{2}{*}{$\begin{array}{l}\text { Relevancy based } \\
\text { on age }\end{array}$} \\
\hline & Seeking & Neutral & Avoiding & & \\
\hline \multicolumn{6}{|l|}{ Anticipated testing decision } \\
\hline Yes (91) & $69(76)$ & $3(3)$ & $9(10)$ & $17(19)$ & $5(5)$ \\
\hline No (18) & $3(17)$ & $10(56)$ & $5(28)$ & $3(17)$ & $5(28)$ \\
\hline \multicolumn{6}{|l|}{ Education } \\
\hline No College (30) & $19(66)$ & $1(3)$ & $1(3)$ & $5(17)$ & $3(10)$ \\
\hline College (82) & $55(67)$ & $13(16)$ & $15(18)$ & $16(20)$ & $7(9)$ \\
\hline \multicolumn{6}{|l|}{ Race/ethnicity } \\
\hline Mexican American (34) & $24(71)$ & $3(9)$ & $3(9)$ & $4(12)$ & $3(9)$ \\
\hline Non-Hispanic Black (39) & $29(74)$ & $5(13)$ & $4(10)$ & $7(18)$ & $2(5)$ \\
\hline Non-Hispanic White (39) & $22(55)$ & $6(15)$ & $9(23)$ & $11(28)$ & $5(13)$ \\
\hline \multicolumn{6}{|l|}{ Perceived T2DM Risk } \\
\hline Low (49) & $30(61)$ & $4(8)$ & $7(14)$ & $8(16)$ & $6(12)$ \\
\hline Average (32) & $23(70)$ & $3(9)$ & $2(6)$ & $9(27)$ & $2(6)$ \\
\hline High (31) & $22(71)$ & $7(23)$ & $7(23)$ & $5(16)$ & $2(6)$ \\
\hline
\end{tabular}

Percentages may not sum to 100 due to rounding or exclusion of missing data. Two participants' testing decisions were classified as "Maybe - neither yes nor no." No testing decision was given by one non-Hispanic White, college-educated participant due to interviewer error. These participants' data are omitted from anticipated testing decision

analysis suggests potential explanations for lower interest in T2DM genetic testing among college-educated and nonHispanic White participants that ought also to be studied. In line with previous research on genetic literacy and education (Christensen et al., 2010; Haga et al., 2013; Lea et al., 2011), college-educated and non-Hispanic White participants were more likely to have expectations for testing that were concordant with current medical science and more frequently expressed concerns or doubts about genetic testing. Taken together, these findings raise important questions that have implications for both health behavior outcomes and tailored patient education. Are patients with higher education more skeptical about the value of T2DM genetic testing because they have a greater understanding for the drawbacks and limitations of genetic testing for complex diseases? Does it follow that historical disparities in education underlie racial/ethnic differences in interest and decision-making processes around T2DM genetic testing? No claims can be made in this study, but these questions deserve further investigation.

\section{Implications for Tailored Patient Education}

Consistent with previous research on genetic literacy (Christensen et al., 2010; Lanie et al., 2004), this study identified several misconceptions about genetic testing that are relevant to patient education and genetic literacy outreach. Participants expressed uncertainty about genetic inheritance, the invasiveness of genetic testing, and the predictive (rather than diagnostic) nature of T2DM genetic test results. Of note, we found that the context of T2DM provides unique opportunities for miscommunication between patients and clinicians about genetic testing. Participants in this study were familiar with routine "diabetes blood tests" (referring to hemoglobin A1c tests or similar). Despite having distinctive purposes, benefits, and risks, routine diabetes testing was sometimes conflated with T2DM genetic testing because both fall into the broad category "blood test." If T2DM genetic testing were to become available to the general public, healthcare providers would be prudent to take special care disentangling more common "diabetes blood tests" from T2DM genetic testing during patient counseling and education.

This study suggests, however, that higher education and higher genetic literacy may actually decrease interest in T2DM genetic testing. While more research is needed, our analysis indicates that individuals with higher education and higher genetic literacy may have a healthy skepticism of the value that genetic testing would add beyond conventional T2DM risk assessments and may be more sensitive to potential harms, such as genetic discrimination. Our findings are in agreement with prior research in which participants with higher genetic knowledge were more likely to perceive DNA testing as "frightening" (Haga et al., 2013). Thus, achieving high genetic literacy should not be the only goal of patient education and counseling. Raising genetic literacy may facilitate weightier conversations between patients and their healthcare providers on the nature of T2DM genetic 
information but may only be the first step toward more informed decision-making around genetic testing.

\section{Implications for Health Behavior Outcomes}

The majority of participants' decision-making centered on their orientation to genetic information (InformationSeeking, Information-Avoiding, or Information-Neutral). Individuals who demonstrated the Information-Seeking theme believed their T2DM genetic test results would motivate them to decrease their personal risk for T2DM (or that of their family members) through lifestyle modification. Research has demonstrated a gulf between intention to change health behaviors and actual behavioral change (Webb \& Sheeran, 2006), and early clinical trials have failed to demonstrate behavioral benefits from T2DM genetic testing in its current limited form (Grant et al., 2013; Voils et al., 2015). Nevertheless, T2DM genetic testing may be beneficial to individuals who are already on the precipice of lifestyle change. In contrast to Information-Seeking participants, InformationAvoiding participants' decision-making process focused on reducing stress, fear, and anxiety over T2DM risk by declining genetic testing. When asked directly, some of these individuals admitted that they already perceived themselves to be at high risk for T2DM, but they also acknowledged that they did not feel ready to make lifestyle changes.

The difference between Information-Seekers and Information-Avoiders may lie in individuals' strategies of coping with stressful experiences (McAndrew et al., 2008). Information-Seeking participants appeared to employ objective-oriented strategies of coping with hypothetical genetic risk (e.g., reducing risk through action), whereas Information-Avoiding participants seemed to rely on psychological coping strategies directed toward regulating their emotional reactions to risk (e.g., denial, avoidance). Thus, interventions that focus first on enhancing positive emotional regulation, such as those derived from Leventhal's Common Sense Model of Self-regulation (Cameron \& Jago, 2008), may help promote healthy behavior changes among those expressing Information-Avoidance toward T2DM genetic testing.

A third distinct group, Information-Neutral participants, saw neither benefit nor harm in T2DM genetic testing. These individuals did not afford any special value to genetic information above other modes of T2DM risk assessment. Thus, most Information-Neutral participants anticipated they would decline genetic testing, and thus their genetic status would have no effect on their behavior.

\section{Strengths and Limitations}

The exploratory approach and purposive sampling in our study limits the generalizability of results and precludes statistical hypothesis testing. Because this study used a hypothetical scenario, participants' anticipated uptake of T2DM genetic testing likely overestimates what the level of uptake would be in a real clinical environment (Persky et al., 2007). Additionally, this study was limited to participants who believed that their genes either increased or decreased their personal risk of T2DM, rather than individuals who believed in a genetic contribution to T2DM in general. Therefore, individuals less open to a genetic contribution to T2DM are underrepresented. Similarly, those who believe genes play a role in T2DM, but do not contribute directly to their own T2DM risk, are underrepresented; however, it is unclear if there would be differences in the interpretation of the interview question by this group of people. Nevertheless, the decision-making process and genetic testing expectations expressed by participants are still highly relevant to pre-test counseling and education.

A number of our findings have importance for future research. First, it is unclear why the trends pertaining to education and interest in genetic testing were only observed in some races/ethnicities; given the qualitative nature of our study, it is inappropriate to draw conclusions from this data. Our findings are of potential interest, however, for hypothesis generation and suggest that hypothesis-based statistical testing in a larger, representative sample may be warranted. Second, although all participants were classified as high-risk for T2DM based on phenotype, $44 \%(n=49)$ of them perceived their risk to be "low." It is unclear whether the combination of phenotypic high risk for T2DM with low risk perception is atypical of the populations sampled. It is possible, though not tested here, that skewed risk perceptions are commonplace. Alternatively, participants were classified as at-risk for T2DM based on selfreported risk factors, rather than medical records. It is unclear whether this procedure over- or underestimated participants' true T2DM risk level (Kuczmarski et al., 2001), an important question for further study.

Finally, we note two important strengths of our research: 1) The exploratory nature of this study allowed participants to volunteer those aspects of T2DM genetic testing that were most meaningful to them, rather than limiting the investigation to predetermined concepts drawn from theory; 2) The inclusion of a large number of participants from diverse demographic backgrounds is a significant addition to the literature, and suggests the need for formally testing group differences using quantitative methods in diverse samples.

\section{Conclusions}

T2DM genetic testing is in its infancy. This study identified factors in the decision-making process and genetic testing expectations that patients from varied backgrounds are likely to bring to such testing protocols. Our findings serve as a foundation to inform genetic counseling and future research on the 
efficacy of T2DM genetic testing. In particular, this study calls for investigation of the effects of educational attainment and racial/ethnic background factors on perceptions about T2DM genetic testing and identifies common misconceptions about the testing process.

Acknowledgments The authors thank Hannah M. Curtis and Alix H. Bernholtz for assistance with qualitative coding, and Ji Qi for help with data management. This project was funded by the National Institute of Diabetes and Digestive and Kidney Diseases of the National Institutes of Health under award number R01DK083347 (to T.E.J.). Additional support was provided by the Michigan Center for Diabetes Translational Research under award number P30DK092926 from the National Institute of Diabetes and Digestive and Kidney Diseases. The content of this publication is solely the responsibility of the authors and does not necessarily represent the official views of the National Institutes of Health.

\section{Compliance with Ethical Standards}

Conflict of Interest Alicia Carmichael, Bailey Hulswit, Emily Moe, Toby Jayaratne, and Beverly Yashar declare that they have no conflict of interest.

Human Studies and Informed Consent All procedures followed were in accordance with the ethical standards of the responsible committee on human experimentation (institutional and national) and with the Helsinki Declaration of 1975, as revised in 2000 (5). Informed consent was obtained from all patients for being included in the study.

Animal Studies No animal studies were carried out by the authors for this article.

\section{References}

Almgren, P., Lehtovirta, M., Isomaa, B., Sarelin, L., Taskinen, M. R., Lyssenko, V., Tuomi, T., \& Groop, L. (2011). Heritability and familiality of type 2 diabetes and related quantitative traits in the Botnia study. Diabetologia, 54, 2811-2819.

American Diabetes Association (2015). Standards of medical care in diabetes-2015. Diabetes Care, 38(Supplement 1), S5-S7.

Becker, F., van El, C. G., Ibarreta, D., Zika, E., Hogarth, S., Borry, P., Cambon-Thomsen, A., Cassiman, J.J., Evers-Kiebooms, G., Hodgson, S., Janssens, A.C., Kaariainen, H., Krawczak, M., Kristoffersson, U., Lubinski, J., Patch, C., Penchaszadeh, V.B., Read, A., Rogowski, W., Sequeiros, J., Tranebjaerg, L., van Langen, I.M., Wallace, H., Zimmern, R., Schmidtke, J., \& Cornel, M.C. (2011). Genetic testing and common disorders in a public health framework: how to assess relevance and possibilities. Background document to the ESHG recommendations on genetic testing and common disorders. European Journal of Human Genetics, 19, S6-S44.

Cameron, L. D., \& Jago, L. (2008). Emotion regulation interventions: a common-sense model approach. British Journal of Health Psychology, 13, 215-221.

Cardona-Morrell, M., Rychetnik, L., Morrell, S. L., Espinel, P. T., \& Bauman, A. (2010). Reduction of diabetes risk in routine clinical practice: are physical activity and nutrition interventions feasible and are the outcomes from reference trials replicable? A systematic review and meta-analysis. BMC Public Health, 10(653), 1-17.
Centre d'Etudes et de Recherche pour l'Intensification du Traitement du Diabète. (2012). Validation of a Predictive Risk Equation for Type 2 Diabetes in Families With Risk (DESCENDANCE). In: ClinicalTrials.gov. Bethesda (MD): - [cited 2016 Feb 2]. Available from: http://clinicaltrials.gov/show/study/NCT01727349 NLM Identifier: NCT01727349.

Christensen, K. D., Jayaratne, T. E., Roberts, J. S., Kardia, S. L., \& Petty, E. M. (2010). Understandings of basic genetics in the United States: results from a national survey of black and white men and women. Public Health Genomics, 13, 467-476.

David Grant U.S. Air Force Medical Center, Duke University. (2013). Genetic risk and health coaching for type 2 diabetes and coronary heart disease. In: ClinicalTrials.gov. Bethesda (MD): - [cited 2016 Feb 2]. Available from: https://clinicaltrials.gov/ct2 /show/study/NCT01884545 NLM Identifier: NCT01884545.

de Miguel-Yanes, J. M., Shrader, P., Pencina, M. J., Fox, C. S., Manning, A. K., Grant, R. W., Dupuis, J., Florez, J. C., D’Agostino, R. B., Cupples, L. A., \& Meigs, J. B. (2011). Genetic risk reclassification for type 2 diabetes by age below or above 50 years using 40 type 2 diabetes risk single nucleotide polymorphisms. Diabetes Care, 34 , 121-125.

Grant, R. W., Hivert, M., Pandiscio, J. C., Florez, J. C., Nathan, D. M., \& Meigs, J. B. (2009). The clinical application of genetic testing in type 2 diabetes: a patient and physician survey. Diabetologia, 52, 2299-2305.

Grant, R. W., O’Brien, K. E., Waxler, J. L., Vassy, J. L., Delahanty, L. M., Bissett, L. G., Green, R. C., Stember, K. G., Guiducci, C., Park, E. R., Florez, J. C., \& Meigs, J. B. (2013). Personalized genetic risk counseling to motivate diabetes prevention: a randomized trial. Diabetes Care, 36, 13-19.

Haga, S. B., Barry, W. T., Mills, R., Ginsburg, G. S., Svetkey, L., Sullivan, J., \& Willard, H. F. (2013). Public knowledge of an attitudes toward genetics and genetic testing. Genetic Testing and Molecular Biomarkers, 17, 327-335.

Hsieh, H. F., \& Shannon, S. E. (2005). Three approaches to qualitative content analysis. Qualitative Health Research, 15, 1277-1288.

International Diabetes Federation. (2013). IDF Diabetes Atlas, 6th edn. Brussels, Belgium: International Diabetes Federation; Retrieved from: http://www.idf.org/diabetesatlas.

Klitzman, R. L. (2010). Misunderstandings concerning genetics among patients confronting genetic disease. Journal of Genetic Counseling, $19,430-446$

Kuczmarski, M. F., Kuczmarski, R. J., \& Najjar, M. (2001). Effects of age on validity of self-reported height, weight, and body mass index: findings from the third National Health and nutrition examination survey, 1988-1994. Journal of the American Dietetic Association, 101, 28-34.

Lanie, A. D., Jayaratne, T. E., Sheldon, J. P., Kardia, S. L., Anderson, E. S., Feldbaum, M., \& Petty, E. M. (2004). Exploring the public understanding of basic genetic concepts. Journal of Genetic Counseling, 13, 305-320.

Lea, D. H., Kaphingst, K. A., Bowen, D., Lipkus, I., \& Hadley, D. W. (2011). Communicating genetic and genomic information: health literacy and numeracy considerations. Public Health Genomics, 14, 279-289.

Lombard, M., Snyder-Duch, J., \& Bracken, C. C. (2002). Content analysis in mass communication: assessment and reporting of intercoder reliability. Human Communication Research, 28, 1468-2958.

MacQueen, K. M., McLellan, E., Kay, K., \& Milstein, B. (1998). Codebook development for team-based qualitative analysis. Field Methods, 10, 31-36.

Markowitz, S. M., Park, E. R., Delahanty, L. M., O’Brien, K. E., \& Grant, R. W. (2011). Perceived impact of diabetes genetic risk testing among patients at high phenotypic risk for type 2 diabetes. Diabetes Care, 34, 568-573. 
McAndrew, L. M., Musumeci-Szabó, T. J., Mora, P. A., Vileikyte, L., Burns, E., Halm, E. A., Leventhal, E. A., \& Leventhal, H. (2008). Using the common sense model to design interventions for the prevention and management of chronic illness threats: From description to process. British Journal of Health Psychology, 12, 195-204.

Meigs, J. B., Shrader, P., Sullivan, L. M., McAteer, J. B., Fox, C. S., Dupuis, J., Manning, A. K., Florez, J. C., Wilson, P. W. F., D'Agostino, R. B., \& Cupples, L. A. (2008). Genotype score in addition to common risk factors for prediction of type 2 diabetes. The New England Journal of Medicine, 359, 2208-2219.

Mills, R., Powell, J., Barry, W., \& Haga, S. B. (2015). Informationseeking and sharing behavior following genomic testing for diabetes risk. Journal of Genetic Counseling, 24, 58-66.

Nishigaki, M., Kobayashi, K., Hitomi, T., Yokomura, T., Yokoyama, M., Seki, N., \& Kazuma, K. (2007). Perception of offspring risk for type 2 diabetes among patients with type 2 diabetes and their adult offspring. Diabetes Care, 30, 3033-3034.

Nishigaki, M., Sato, E., Ochiai, R., Shibayama, T., \& Kazuma, K. (2011). Impact of a booklet about diabetes genetic susceptibility and its prevention on attitudes towards prevention and perceived behavioral change in patients with type 2 diabetes and their offspring. Advances in Preventive Medicine, 2011, 1-7.

Nishigaki, M., Tokunaga-Nakawatase, Y., Nishida, J., \& Kazuma, K. (2014). The effect of genetic counseling for adult offspring of patients with type 2 diabetes on attitudes toward diabetes and its heredity: a randomized controlled trial. Journal of Genetic Counseling, 23, 762-769.

O'Daniel, J. M. (2010). The prospect of genome-guided preventive medicine: a need and opportunity for genetic counselors. Journal of Genetic Counseling, 19, 315-327.

Persky, S., Kaphingst, K. A., Condit, C. M., \& McBride, C. M. (2007). Assessing hypothetical scenario methodology in genetic susceptibility testing analog studies: a quantitative review. Genetics in Medicine, 9, 727-738.

Proove Bioscience, Inc. (2015). A Study of the Impact of Genetic Testing on Clinical Decision Making and Patient Care (REVOLUTION). In: ClinicalTrials.gov. Bethesda (MD): - [cited 2016 Feb 2]. Available from: http://clinicaltrials.gov/show/study/NCT02487888 NLM Identifier: NCT02487888.

Schellenberg, E. S., Dryden, D. M., Vandermeer, B., Ha, C., \& Korownyk, C. (2013). Lifestyle interventions for patients with and at risk for type 2 diabetes: a systematic review and meta-analysis. Annals of Internal Medicine, 159, 543-551.

Tessaro, I., Smith, S. L., \& Rye, S. (2005). Knowledge and perceptions of diabetes in an Appalachian population. Preventing Chronic Disease, 2(2), 1-9.

U.S. Department of Health and Human Services. (2008). 2008 Physical Activity Guidelines for Americans. (Report No. U0036). Retrieved from http://www.health.gov/paguidelines/pdf/paguide.pdf.

Vassy, J. L., Donelan, K., Hivert, M. F., Green, R. C., \& Grant, R. W. (2013). Genetic susceptibility testing for chronic disease and intention for behavior change in healthy young adults. Journal of Community Genetics, 4, 263-271.

Vassy, J. L., O’Brien, K. E., Waxler, J. L., Park, E. R., Delahanty, L. M., Florez, J. C., Meigs, J. B., \& Grant, R. W. (2012). Impact of literacy and numeracy on motivation for behavior change after diabetes genetic risk testing. Medical Decision Making, 32, 606-615.

Voils, C. I., Coffman, C. J., Grubber, J., Edelman, D., Sadeghpour, A., Maciejewski, M. L., Bolton, J., Cho, A., Ginsburg, G. S., \& Yancy Jr., W. S. (2015). Does type 2 diabetes genetic testing and counseling reduce modifiable risk factors? A randomized controlled trial of veterans. Journal of General Internal Medicine, 30, 1591-1598.

Waxler, J. L., O’Brien, K. E., Delahanty, L. M., Meigs, J. B., Florez, J. C., Park, E. R., Pober, B. R., \& Grant, R. W. (2012). Genetic counseling as a tool for type 2 diabetes prevention: a genetic counseling framework for common polygenetic disorders. Journal of Genetic Counseling, 21, 684-691.

Webb, T. L., \& Sheeran, P. (2006). Does changing behavioral intentions engender bahaviour change? A meta-analysis of the experimental evidence. Psychological Bulletin, 132, 249-268.

WHO Department of Health Statistics and Information Systems. (2013). Global health estimates summary tables: YLLs by cause, Age, and sex. In: GHE_YLL_Global_2000_2011.xls, editor. Geneva, Switzerland: World Health Organization.

Wright, C. F., \& Kroese, M. (2009). Evaluation of genetic tests for susceptibility to common complex diseases: why, when and how? Human Genetics, 127, 125-134.

Yates, T., Davies, M., \& Khunti, K. (2009). Preventing type 2 diabetes: Can we make the evidence work? Postgraduate Medicine Journal, $85,475-480$. 